\title{
Image database, Al and capsule endoscopy; the bets are on
}

다 (i)

\author{
Authors \\ Anastasios Koulaouzidis ${ }^{1}$, Ervin Toth ${ }^{2}$ \\ Institutions \\ 1 Endoscopy Unit, Centre for Liver \& Digestive Disorders, \\ The Royal Infirmary of Edinburgh, Scotland \\ 2 Department of Gastroenterology, Skåne University \\ Hospital, Malmö, Lund University, Sweden \\ Bibliography \\ DOI https://doi.org/10.1055/a-1073-7726 | \\ Endoscopy International Open 2020; 08: E421-E422
}

Capsule has gotten its time certification to become the main endoscopic modality for small bowel inspection. Capsule endoscopy (CE) therefore has become one more technique for the millennial generation that now look at the future with strength, will and determination to go down more disruptive paths [1]. Nevertheless, even for courageous youth, maturing comes with certain "obligations" and societal requirements. Of course, when medical technology is the subject in focus, this translates more or less into diagnostic yield, complication rates, and quality/performance assurance measures [2-4].

Moreover, in terms of open issues in the field, there are still a few. Perhaps we have little hesitation in counting the indications for CE in a single breath or even providing similar complication rates and pathways for safe "capsuling," but for those of us who read CE videos in high-volume centers, there are several technological tweaks that we wish the manufacturers would have sorted by now. They range from better image resolution and battery lifetime to various software enhancements and/or hardware advancement(s) [5,6].

In fact, we kept repeating the cry for the next TIDAL wave in CE revolution, i.e. one that will include Therapy capacity, Integration/Intelligence, Data (not only images) collection, Actuation, and Localization summarizing the main challenges that any - of the many currently available - CE provision systems has to face to increase its market proposal and value over its competitors. The buzz of artificial intelligence ( $\mathrm{Al}$ ) is not new; the very essence of CE reading software, in fact, is one of early $\mathrm{Al}$ in action. Tools such as Lewis Score [7], QuickView [8], Suspected Blood Indicator [9] are nothing more than clever snippets of Al integrated in the very early versions of proprietary reading software with main aim to assist, support and/or speed up medical decision process [10].
However, errors and oversights are akin to human nature; when these are associated with erroneous diagnosis, the impact/harm for both patients and healthcare professionals who experience them may be detrimental and associated with loss, emotional hardship, and litigation. On the other hand, striving for improvement is at the heart of all integrated, people-centered health care systems, although that can be a challenging and often slow process. In CE, just as with other imaging specialties such as radiology in which the record of each examination is readily available at any time for review and comparison, the ground is fertile for application of Al to limit findings miss rate, reading time and/or aid lesion characterization and decision-making via computer-aided diagnosis (CAD).

In conventional endoscopy/colonoscopy, systems such as GI Genius, ENDOANGEL and OdinVision are making impact trial after trial but they are all based on/assisted by the human operator in detection of lesions. Nevertheless, CE has the advantage of existing repositories of imaging studies and of course that of "the cloud." Our deep belief is that the CE should be interpreted on board $[11,12]$ just like a modern electrocardiogram of the bowels. In 2017, we reported that incorporating machine learning algorithms (MLAs) into CE reading is difficult as large amounts of image annotations are required for training. We had noted back then, presenting KID (a CE interactive database), that existing databases lack graphic annotations of pathologies and cannot be used for advancement of software solutions, maybe the correct term should have been AI [13].

It's our pleasure to see that Leenhardt et al have been extremely busy recently structuring a framework for a big, national multicentre repository of small bowel CE images [14]. The images have been obtained, for the purpose of clinical care, with PillCamSB3, (Medtronic, Minnesota, United States) from 12 
participating centers in France. The study met the approval of the French Data Protection Authority. In total, more than 4,000 CE pooled studies were reviewed and an impressive 6,013 still frames and their respective short video clips were collected. Applying some crowd sourcing, Leenhardt et al used medical students to delineate (annotate) abnormalities in 5,124 frames which was then reviewed by CE experts. This database was called CAD-CAP (Computer-Assisted Diagnosis for CAPsule endoscopy). CAD-CAP is certainly the largest PillCamSB3 database in existence. However, even this database, as the author mentioned, is dwarfed by relevant attempts in ophthalmology and dermatology. The same active group recently presented the use of convolutional neural networks in detection of small bowel angiectasias utilizing this database and therefore proving its research-support potential [15]. Prof Dray's colonic computed assessment of cleansing (CAC) score was able, based on the ratio of color intensities, to discriminate "adequately cleansed" from "inadequately cleansed" CCE still frames [16]. Furthermore, the very same team is spearheading another major challenge of the times, an attempt for unification of nomenclature of lesions in CE [17].

Essentially, we all aspire that in a few years we will be able to rely on $\mathrm{Al}$ to analyze $\mathrm{CE}$ videos and present us with its findings. Multiple research and commercial entities across the world are working on respective systems training neural networks. In the meantime, it is certainly beneficial to have efforts and works like those of Leenhardt et al to foster essential multidisciplinary cooperation and make progress in this field. For this reason, we trust that the team will make the images and their annotations available to the wider scientific community and continue with the hard work required to continue populating the database with polyps images and relevant annotations.

\section{Competing interests}

AK is cofounder of AJMMedicaps, has received travel support and lecture honoraria from Jinshan, travel support and material support for research from SynMed and IntroMedic, and advisory board for IntroMedic, he has also been advisory board for Dr FalkPharmaUk, Tillots and received lecture honoraria from Dr FalkPharmaUK, and Ferring. ET received honoraria and/or travel support from Olympus, Medtronic, Norgine and Ambu. ee

\section{References}

[1] Watts ]. The Greta Thunberg effect: At last MPs focus on climate change. The Guardian; 2019: https://www.theguardian.com/environment/2019/apr/23/greta-thunberg
[2] Koulaouzidis A, Rondonotti E, Karargyris A. Small-bowel capsule endoscopy: a ten-point contemporary review. World J Gastroenterol 2013; 19: 3726-3746

[3] Rondonotti E, Spada C, Adler S et al. Small-bowel capsule endoscopy and device-assisted enteroscopy for diagnosis and treatment of small-bowel disorders: European Society of Gastrointestinal Endoscopy (ESGE) Technical Review. Endoscopy 2018; 50: 423-446

[4] Spada C, McNamara D, Despott EJ et al. Performance measures for small-bowel endoscopy: A European Society of Gastrointestinal Endoscopy (ESGE) Quality Improvement Initiative. United European Gastroenterol J 2019; 7: 614-641

[5] Vasilakakis M, Koulaouzidis A, Yung DE et al. Follow-up on: optimizing lesion detection in small bowel capsule endoscopy and beyond: from present problems to future solutions. Expert Rev Gastroenterol Hepatol 2019; 13: 129-141

[6] lakovidis DK, Koulaouzidis A. Software for enhanced video capsule endoscopy: challenges for essential progress. Nat Rev Gastroenterol Hepatol 2015; 12: 172-186

[7] Yablecovitch D, Lahat A, Neuman S et al. The Lewis score or the capsule endoscopy Crohn's disease activity index: which one is better for the assessment of small bowel inflammation in established Crohn's disease? Therap Adv Gastroenterol 2018: doi:10.1177| $1756283 \times 17747780$

[8] Koulaouzidis A, Smirnidis A, Douglas S et al. QuickView in small-bowel capsule endoscopy is useful in certain clinical settings, but QuickView with Blue Mode is of no additional benefit. Eur J Gastroenterol Hepatol 2012; 24: 1099-1104

[9] Yung DE, Sykes C, Koulaouzidis A. The validity of suspected blood indicator software in capsule endoscopy: a systematic review and metaanalysis. Expert Rev Gastroenterol Hepatol 2017; 11: 43-51

[10] lakovidis DK, Koulaouzidis A. Automatic lesion detection in capsule endoscopy based on color saliency: closer to an essential adjunct for reviewing software. Gastrointest Endosc 2014; 80: 877-883

[11] Koulaouzidis A, lakovidis DK, Karargyris A et al. Wireless endoscopy in 2020: Will it still be a capsule? World J Gastroenterol 2015; 21: 51195130

[12] NAutILES project. https://nautilesorg.wordpress.com

[13] Koulaouzidis A, lakovidis DK, Yung DE et al. KID Project: an internetbased digital video atlas of capsule endoscopy for research purposes. Endosc Int Open 2017; 5: E477-E483

[14] Leenhardt R, Li C, Le MouelJP et al. CAD-CAP: A 25000-image database serving the development of artificial intelligence for capsule endoscopy. Endosc Int Open 2020; 08: E415-E420

[15] Leenhardt R, Vasseur P, Li C. CAD-CAP Database Working Group. et al. A neural network algorithm for detection of $\mathrm{Gl}$ angiectasia during small-bowel capsule endoscopy. Gastrointest Endosc 2019; 89: 189194

[16] Becq A, Histace A, Camus M et al. Development of a computed cleansing score to assess quality of bowel preparation in colon capsule endoscopy. Endosc Int Open 2018; 6: E844-E850

[17] Leenhardt R, Li C, Koulaouzidis A et al. Nomenclature and semantic description of vascular lesions in small bowel capsule endoscopy: an international Delphi consensus statement. Endosc Int Open 2019; 7: E372-E379 